International Journal of Engineering \& Technology, $7(2.23)(2018) 47-56$
International Journal of Engineering \& Technology
SPC
Website: $w$ ww.sciencepubco.com/index.php/IJET
Research paper

\title{
Nonlinear Vibration of Microbeams Based on The Elastics Foundation Using High-Order Energy Balance Method And Global Error Minimization Method
}

\author{
D. V. Hieu \\ Department of Mechanics, Thai Nguyen University of Technology, Thai Nguyen, Viet Nam \\ E-mail: hieudv@tnut.edu.vn
}

\begin{abstract}
In this paper, nonlinear vibration of microbeams based on the nonlinear elastic foundation is investigated. The equation of motion of microbeams based on three-layered nonlinear elastic medium (shear, linear and nonlinear layers) is described by the partial differential equation by using the modified couple stress theory. The equation of motion of microbeams is transformed into the ordinary differential equation by using Galerkin method. The high-order Energy Balance method and the high-order Global Error Minimization method are used to get the frequency - amplitude relationships for the nonlinear vibration of microbeams with pinned-pinned and clampedclamped end conditions. Comparisons between the present solutions and the privious solutions show the accuracy of the obtained results.
\end{abstract}

Keywords: Microbeams, nonlinear vibration, Energy Balance method, Global Error Minimization method.

\section{Introduction}

Micro- and nano-beams have been widely used in nano- and micro-sized systems and devices such as biosensors, nanowires, atomic force microscope, microactuators, nano-probes, microelectromechanical, ultra thin films and nano-electromechanical systems. In these applications, the size effect has a major role on static and dynamic deformation behavior of material and cannot be negligible [1,2]. The classical continuum mechanics is not able to take into account the size effect in modeling of the material behavior at the nanoscale. Thus, several higher order continuum theories, such as the couple stress theory $[3,4,5]$ that contains four material constants (two classical and two additional), strain gradient theory [6], micropolar theory [7], nonlocal elasticity theory [8], surface elasticity [9], have been developed to account for the size effect in the small scale structures. The classical couple stress theory is one of the other higher order continuum theories which contain two additional material length scale parameters besides the classical constants for an isotropic elastic material, elaborated by Mindlin and Tiersten [4], Toupin [3]. A modified couple stress theory was proposed by Yang et al. [10] which contain only one additional material length scale parameter in addition to the classical material constants. Also, the couple stress tensor is symmetric in this theory, this modified couple stress theory includes the secondorder displacement gradients. This modified couple stress theory is more useful than classical one due to these features.

To date, static, buckling and vibration characteristics of microbeams using the modified couple stress theory have been theo- retically investigated in the context of size-dependent beam theories by the several researchers $[11,12,13,14,15,16,17]$.

Recently, nonlinear static and free vibration analysis of microbeams based on the nonlinear elastic foundation using modified couple stress theory are studied by Simsek [18]. In this work, the approximate analytical expressions of nonlinear frequency of microbeams based on the nonlinear foundation are obtained and presented for the first time for pinned-pinned (P-P) and clampedclamped (C-C) end conditions by means of He's Variational Approach (VA). Static analysis is also performed for uniformly distributed load.

Due to the limitation of the exact solution of nonlinear oscillation problems, new asymptotic methods have been increasingly developed in order to solve nonlinear differential equations such as Energy Balance method (EBM) [19], Variational Approach (VA) [20], Hamiltonian Approach (HA) [21], Global Error Minimization method (GEM) [22]. To improve the approximate solution of nonlinear oscillators, the high-order approximate methods are introducted such as the high-order Energy Balance method [23], the high-order Global Error Minimization method [22].

In this paper, the high-order Energy Balance method and the highorder Global Error Minimization method will be appied to analyse nonlinear free vibration of microbeams based on nonlinear elastic foundation. The obtained solutions are compared with the ones achived by Simsek using He's Variational Approach. Comparisons show the accuracy of the present solutions. 


\section{Modelling and formulation}

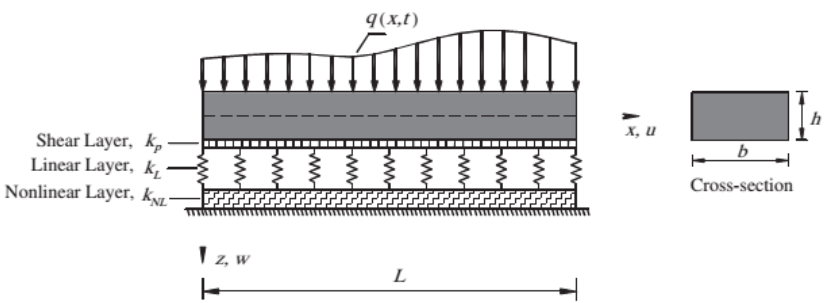

Fig. 1: An isotropic microbeam based on a three-layered nonlinear elastic foundation.

An isotropic microbeam of a constant thickness of $h$ with crosssectional dimensions $L$ and $b$ is considered and shown in Fig. 1 . The microbeam is based on a nonlinear elastic foundation with the spring constants $k_{L}, k P$ and $k_{N L}$ of the Winkler elastic medium, Pasternak elastic medium and nonlinear elastic medium, respectively.

Using the modified couple theory, the equations of motion in terms of the displacements can be achieved as follows [18]:

$$
\begin{aligned}
& \left(E I+\mu A L^{2}\right) \frac{\partial^{4} w}{\partial x^{4}}-\left[\frac{E A}{2 L} \int_{0}^{L}\left(\frac{\partial w}{\partial x}\right)^{2} d x\right] \frac{\partial^{2} w}{\partial x^{2}} \\
& +k_{L} w-k_{P} \frac{\partial^{2} w}{\partial x^{2}}+k_{N L} w^{3}+\rho A \frac{\partial^{2} w}{\partial t^{2}}=q(x, t)
\end{aligned}
$$

where $N$ is the axial normal force, $E$ is the elasticity modulus, $A$ is the area of the cross-section, $I$ is the inertia moment of the crosssection, $\mu=E / 2(1+v)$ is known as shear modulus where $v$ is the Poisson's ratio, $\rho$ is density, $q$ is the distributed transverse load. The following dimensionless parameters are introduced:

$$
\begin{aligned}
& \bar{x}=\frac{x}{L}, \bar{w}=\frac{w}{r}, t=\bar{t} \sqrt{\frac{\rho A L^{4}}{E I}}, \eta=\frac{l}{h}, S=1+\frac{6 \eta^{2}}{1+v}, \\
& K_{L}=\frac{k_{L} L^{4}}{E I}, K_{P}=\frac{k_{P} L^{2}}{E I}, K_{N L}=\frac{k_{N L} r^{2} L^{4}}{E I}, \bar{q}=\frac{q L^{4}}{E I r} .
\end{aligned}
$$

where $r=\sqrt{I / A}$ is the radius of gyration of the cross-section, and it is noted that $l$ denotes the material length scale parameter which is an additional material parameter enabling the theory to capture the size-dependency. Considering Eq. (2), Eq. (1) can be written in the non-dimensional form as:

$$
S \frac{\partial^{4} \bar{w}}{\partial \bar{x}^{4}}-\left[\frac{1}{2} \int_{0}^{1}\left(\frac{\partial \bar{w}}{\partial \bar{x}}\right)^{2} d \bar{x}\right] \frac{\partial^{2} \bar{w}}{\partial \bar{x}^{2}}+K_{L} \bar{w}-K_{P} \frac{\partial^{2} \bar{w}}{\partial \bar{x}^{2}}+K_{N L} \bar{w}^{3}+\frac{\partial^{2} \bar{w}}{\partial \bar{t}^{2}}=\bar{q}
$$

In order to apply the Galerkin method, displacement function $\bar{w}(\bar{x}, \bar{t})$ can be expanded into a finite series as follows:

$$
\bar{w}(\bar{x}, \bar{t})=Q(\bar{t}) \phi(\bar{x})
$$

where $Q(\bar{t})$ is the unknown time-dependent coefficient to be determined and $\phi(\bar{x})$ is the basis (test) function which must satisfy the kinematic boundary conditions. The following test functions can be chosen for PP and CC boundary conditions: for Pinned - Pinned (P-P) microbeam:

$$
\phi(\bar{x})=\sin (\pi \bar{x})
$$

for Clamped - Clamped microbeam:

$$
\phi(\bar{x})=\frac{1}{2}[1-\cos (2 \pi \bar{x})]
$$

where $\phi(\bar{x})$ is normalized in such a way that $\phi(0.5)=1$. Substituting the approximate solution in Eq.(4) into Eq.(3), then multiplying both sides of the resulting equation with $\phi(\bar{x})$ and integrating it over the domain $(0,1)$ yields:

$$
\ddot{Q}(\bar{t})+\left(D_{1}+D_{2}+K_{L}\right) Q(\bar{t})+\left(D_{3}+D_{4}\right) Q^{3}(\bar{t})=F(\bar{t})
$$

Here the coefficients $D_{1}, D_{2}, D_{3}, D_{4}$ and $F$ in Eq. (7) can be expressed as:

$$
\begin{gathered}
D_{1}=\frac{\int_{0}^{1} S \phi^{(4)} \phi d \bar{x}}{\int_{0}^{1}(\phi)^{2} d \bar{x}}, D_{2}=-\frac{\int_{0}^{1} K_{P} \phi^{\prime \prime} \phi d \bar{x}}{\int_{0}^{1}(\phi)^{2} d \bar{x}}, D_{3}=-\frac{\frac{1}{2} \int_{0}^{1}\left(\phi^{\prime}\right)^{2} d \bar{x} \int_{0}^{1} \phi^{\prime \prime} \phi d \bar{x}}{\int_{0}^{1}(\phi)^{2} d \bar{x}}, \\
D_{4}=\frac{\int_{0}^{1} K_{N L}(\phi)^{4} d \bar{x}}{\int_{0}^{1}(\phi)^{2} d \bar{x}}, F=\frac{\int_{0}^{1} \bar{q} \phi d \bar{x}}{\int_{0}^{1}(\phi)^{2} d \bar{x}}
\end{gathered}
$$

where $\phi^{(4)}$ is the fourth derivative of $\phi$ with respect to the axial coordinate $\bar{x}$, and the prime notations also denote the differentiations with respect to the axial coordinate.

\section{Analyzing of nonlinear free vibration}

The microbeam is subjected to the following initial conditions:

$$
Q(0)=\alpha, \dot{Q}(0)=0
$$

where $\alpha=w_{\max }(0.5) / r$ is the dimensionless maximum vibration amplitude of the microbeam.

By omitting the forcing term $F$ for free vibration analysis, Eq. (7) can be rewritten in a compact form as follows:

$$
\ddot{Q}(\bar{t})+g_{1} Q(\bar{t})+g_{2} Q^{3}(\bar{t})=0
$$

where $g_{1}=D_{1}+D_{2}+K_{L}$ and $g_{2}=D_{3}+D_{4}$.

In the next section, the high-order Energy Balance method and the high-order Global Error Minimization method will be applied to find approximate solutions of Eq. (10) with the initial conditions (9).

\subsection{Application of the high-order Energy Balance method}

\subsubsection{The first-order approximate}

The basic of the energy balance method (EBM) was proposed by $\mathrm{He}$ [19].The variational of Eq. (10) is given as follows:

$$
J(Q)=\int_{0}^{T / 4}\left(-\frac{\dot{Q}^{2}}{2}+g_{1} \frac{Q^{2}}{2}+g_{2} \frac{Q^{4}}{4}\right) d \bar{t}
$$

Here $T=2 \pi / \omega$ is the period of the nonlinear oscillator.

For the first-order approximation, the following approximate solution of Eq. (10) can be assumed:

$$
Q_{1}(\bar{t})=\alpha \cos (\omega \bar{t})
$$


where $\alpha$ and $\omega$ are the initial amplitude and natural frequency of the nonlinear oscillator, respectively.

With the approximate solution (12), the Hamiltonian of Eq. (10) is given as follows:

$$
\begin{aligned}
H_{1}=\frac{1}{2} \alpha^{2} \omega^{2} \sin ^{2}(\omega \bar{t})+ & \frac{1}{2} g_{1} \alpha^{2} \cos ^{2}(\omega \bar{t})+\cos ^{4}(\omega \bar{t})= \\
& =\frac{1}{2} g_{1} \alpha^{2}+\frac{1}{4} g_{2} \alpha^{4}
\end{aligned}
$$

Eq. (13) yields the following residual equation:

$$
\begin{gathered}
R_{1}(\bar{t})=\frac{1}{2} \alpha^{2} \omega^{2} \sin ^{2}(\omega \bar{t})+\frac{1}{2} g_{1} \alpha^{2} \cos ^{2}(\omega \bar{t})+\cos ^{4}(\omega \bar{t})- \\
-\frac{1}{2} g_{1} \alpha^{2}-\frac{1}{4} g_{2} \alpha^{4} .
\end{gathered}
$$

If, by chance, the exact solution of Eq. (10) had been chosen as the trial function, then it would be possible to make $R_{l}$ zero for all values of $\bar{t}$ by appropriate choice of $\omega$. Since Eq. (12) is only an approximation to the exact solution, $R_{l}$ can not be made zero everywhere. Collocation at $\omega \bar{t}=\pi / 4$, from Eq. (14) we obtain the first-order approximate frequency:

$$
\omega_{E B M 1}=\sqrt{g_{1}+\frac{3}{4} g_{2} \alpha^{2}}
$$

\subsubsection{The high-order approximate}

In order to improve the accuracy of the Energy Balance method, let us define $Q(\bar{t})$ as follows:

$$
Q_{2}(\bar{t})=\alpha_{1} \cos (\omega \bar{t})+\alpha_{2} \cos (3 \omega \bar{t})
$$

Eq. (16) must satisfy the initial conditions (9); therefore, we have:

$$
\alpha_{1}+\alpha_{2}=\alpha
$$

Thus, the approximate solution (16) can be rewritten:

$$
Q_{2}(\bar{t})=\alpha_{1} \cos (\omega \bar{t})+\left(\alpha-\alpha_{1}\right) \cos (3 \omega \bar{t})
$$

With the approximate solution (18), we have the following residual equation:

$$
\begin{aligned}
R_{2}(\bar{t}) & =\frac{1}{2}\left[-\alpha_{1} \omega \sin (\omega \bar{t})-3\left(\alpha-\alpha_{1}\right) \omega \sin (3 \omega \bar{t})\right]^{2} \\
& +\frac{1}{2} g_{1}\left[\alpha_{1} \cos (\omega \bar{t})+3\left(\alpha-\alpha_{1}\right) \cos (3 \omega \bar{t})\right]^{2} \\
& +\frac{1}{4} g_{2}\left[\alpha_{1} \cos (\omega \bar{t})+3\left(\alpha-\alpha_{1}\right) \cos (3 \omega \bar{t})\right]^{4} \\
& -\frac{1}{2} g_{1} \alpha^{2}-\frac{1}{4} g_{2} \alpha^{4}
\end{aligned}
$$

It requires an system of two equations to find two unknown parameters $A_{l}$ and $\omega$. Using Galerkin method as a weighting function, with $\cos (\omega \bar{t})$ and $\cos (3 \omega \bar{t})$ are used as weighting functions, the following systems can be obtained [23]:

$$
\begin{aligned}
& \int_{0}^{T / 4} R_{2}(\bar{t}) \cos (\omega \bar{t}) d \bar{t}=0 \\
& \int_{0}^{T / 4} R_{2}(\bar{t}) \cos (3 \omega \bar{t}) d \bar{t}=0
\end{aligned}
$$

By inserting Eq. (19) into Eqs. (20) and (21), after some mathematical amendments, we have:

$$
\begin{aligned}
& -\frac{75776}{4095} g_{2} \alpha_{1}+\frac{16}{35} g_{1} \alpha_{1}^{2}-\frac{10240}{3003} g_{2} \alpha_{1}^{3}+\frac{512}{1287} g_{2} \alpha_{1}^{4} \\
& -\frac{32}{21} g_{1} \alpha_{1}-\frac{528}{35} \alpha_{1} \omega^{2}+\frac{184}{105} \alpha_{1}^{2} \omega^{2}+\frac{1224}{35} \omega^{2} \\
& +\frac{62464}{5005} g_{2} \alpha_{1}^{2}-\frac{136}{35} g_{1}-\frac{195904}{5005} g_{2}=0 . \\
& \frac{1632256}{45045} g_{2} \alpha_{1}-\frac{176}{315} g_{1} \alpha_{1}^{2}+\frac{22528}{4095} g_{2} \alpha_{1}^{3} \\
& -\frac{3584}{6435} g_{2} \alpha_{1}^{4}+\frac{928}{315} g_{1} \alpha_{1}+\frac{176}{35} \alpha_{1} \omega^{2} \\
& -\frac{104}{105} \alpha_{1}^{2} \omega^{2}-8 \omega^{2}-\frac{44032}{2145} g_{2} \alpha_{1}^{2}+\frac{8}{9} g_{1}+\frac{448}{45} g_{2}=0 .
\end{aligned}
$$

By solving Eqs. (22) and (23) simultaneously, we can obtain the second-order approximate amplitude-frequency relationship.

\subsection{Application of the high-order Global Error Minimi- zation method}

A modified variational approach called Global Error Minimization (GEM) method was introduced by Yadollah Farzaneh and Ali Akbarzadeh Tootoonchi [22]. In this section, we will apply this method to find the approximate solution of Eq. (10).

\subsubsection{The first-order approximate}

It is similar to the Energy Balance method, with the first-order approximate, we begin the procedure with the simplest trial solution:

$$
Q_{1}(\bar{t})=\alpha \cos (\omega \bar{t})
$$

Next, we convert Eq. (10) to the minimization problem:

$$
\begin{array}{ll}
\text { Minimize } & E\left(\dot{Q}_{1}, Q_{1}, \bar{t}\right)=\int_{0}^{T}\left(\ddot{Q}_{1}+g_{1} Q_{1}+g_{2} Q_{1}^{3}\right)^{2} d \bar{t}, T=\frac{2 \pi}{\omega} \\
\text { s.t. } & Q_{1}(0)=\alpha, \quad \dot{Q}_{1}(0)=0
\end{array}
$$

By replacing Eq. (24) in Eq. (25) and performing the integration we get:

Minimize $E\left(\dot{Q}_{1}, Q_{1}, \bar{t}\right)=\frac{\alpha^{2} \pi\left(\begin{array}{l}5 g_{2}^{2} \alpha^{4}-16 \omega^{2} g_{1}-12 \omega^{2} g_{2} \alpha^{2} \\ +8 \omega^{4}+12 g_{1} g_{2} \alpha^{2}+8 g_{1}^{2}\end{array}\right)}{8 \omega}$

Thus, from the condition

$$
\frac{\partial E\left(\dot{Q}_{1}, Q_{1}, \bar{t}\right)}{\partial \omega}=0
$$

we get the first-order approximate frequency of the oscillator:

$$
\omega_{G E M 1}=\frac{1}{6} \sqrt{9 g_{2} \alpha^{2}+12 g_{1}+3 \sqrt{39 g_{2}^{2} \alpha^{4}+96 g_{1} g_{2} \alpha^{2}+64 g_{1}^{2}}}
$$

\subsubsection{The high-order approximate}

Now, to improve our analytical approximation, we will add additional terms to the trial solution by considering the second-order approximation:

$$
Q_{2}(\bar{t})=\alpha_{1} \cos (\omega \bar{t})+\left(\alpha-\alpha_{1}\right) \cos (3 \omega \bar{t})
$$

With the new trial solution in Eq. (28), Eq. (10) is converted to a minimization problem: 


$$
\begin{array}{ll}
\text { Minimize } & E\left(\dot{Q}_{2}, Q_{2}, \bar{t}\right)=\int_{0}^{T}\left(\ddot{Q}_{2}+g_{1} Q_{2}+g_{2} Q_{2}^{3}\right)^{2} d \bar{t}, T=\frac{2 \pi}{\omega} \\
\text { s.t. } & Q_{2}(0)=\alpha, \quad \dot{Q}_{2}(0)=0
\end{array}
$$

The solution of Eq. (29) could be found by using the conditions:

$$
\frac{\partial E\left(\dot{Q}_{2}, Q_{2}, \bar{t}\right)}{\partial \omega}=\frac{\partial E\left(\dot{Q}_{2}, Q_{2}, \bar{t}\right)}{\partial \alpha_{1}}=0
$$

Substituting Eq. (28) into Eq. (29), and from the conditions (30), after some mathematical operations, we get:

$$
\begin{aligned}
& 31488 \alpha_{1}^{2} \omega^{4}-622080 \omega^{4} \alpha_{1}-256 g_{1}^{2} \alpha_{1}^{2}+2560 g_{1}^{2} \alpha_{1} \\
& -880 g_{2}^{2} \alpha_{1}^{6}+4000000 g_{2}^{2} \alpha_{1}^{3}+31200 g_{2}^{2} \alpha_{1}^{5} \\
& -480000 g_{2}^{2} \alpha_{1}^{4}+48000000 g_{2}^{2} \alpha_{1}-19200000 g_{2}^{2} \alpha_{1}^{2} \\
& -2560 \alpha_{1}^{2} \omega^{2} g_{1}-4992 \alpha_{1}^{4} \omega^{2} g_{2}+138240 \alpha_{1}^{3} \omega^{2} g_{2} \\
& -1420800 \alpha_{1}^{2} \omega^{2} g_{2}+46080 \alpha_{1} \omega^{2} g_{1}+6912000 \alpha_{1} \omega^{2} g_{2} \\
& +768000 g_{1} g_{2} \alpha_{1}+3110400 \omega^{4}-230400 \omega^{2} g_{1} \\
& -17280000 \omega^{2} g_{2}-12800 g_{1}^{2}-1920000 g_{1} g_{2} \\
& -80000000 g_{2}^{2}=0 . \\
& 20992 \omega^{4} \alpha_{1}-207360 \omega^{4}+512 g_{1}^{2} \alpha_{1}-2560 g_{1}^{2} \\
& +5280 g_{2}^{2} \alpha_{1}^{5}-12000000 g_{2}^{2} \alpha_{1}^{2}-156000 g_{2}^{2} \alpha_{1}^{4} \\
& +1920000 g_{2}^{2} \alpha_{1}^{3}-48000000 g_{2}^{2}+38400000 g_{2}^{2} \alpha_{1} \\
& -5120 \omega^{2} \alpha_{1} g_{1}-19968 \alpha_{1}^{3} \omega^{2} g_{2}+414720 \alpha_{1}^{2} \omega^{2} g_{2} \\
& -2841600 \alpha_{1} \omega^{2} g_{2}+46080 \omega^{2} g_{1}+6912000 \omega^{2} g_{2} \\
& +3584 g_{1} \alpha_{1}^{3} g_{2}-61440 g_{1} \alpha_{1}^{2} g_{2}+384000 g_{1} \alpha_{1} g_{2} \\
& -768000 g_{1} g_{2}=0 .
\end{aligned}
$$

Eqs. (31) and (32) contain two unknown parameters $\omega$ and $\alpha_{1}$. By solving Eqs. (31) and (32) simultaneously, we can obtain the second-order approximate amplitude-frequency relationship of the oscillator.

Higher-order approximate solutions of the EBM and GEM can be established by the similar manner. However, with higher-order approximations, it is required to solve a complex set of algebraic equations to achieve the frequency of the oscillator.

\section{Numerical results}

The nonlinear frequencies of P-P and C-C Microbeams obtained in this work are compared with the ones achived by Simsek [18]. In this work, using He's Variational Approach (VA), Simsek obtained the nonlinear frequencies as follows:

$$
\omega_{V A}=\sqrt{g_{1}+\frac{3}{4} g_{2} \alpha^{2}}
$$

It can be seen that the appriximate frequency $\omega_{V A}$ in Eq. (33) is the same as the approximate frequency obtained by the firt-order Energy Balance method in Eq. (15). Tables 1 and $\mathbf{2}$ show comparions the approximate frequencies with the exact frequencies for $\mathrm{P}$ $\mathrm{P}$ and C-C Microbeams, respectivelly. Note that these comparisons are calculated with $K_{L}=0, K_{P}=0, K_{N L}=0$ and length scale parameter $l=0$. The exact frequency of the oscillator can be seen Appendix. From these Tables, the accuaracy of the present solutions can be observed.

Table 1: Comparion of the approximate frequencies for P-P Microbeam

\begin{tabular}{ccccccc}
\hline$\alpha$ & $\omega_{e x}$ & $\omega_{V A}($ error $\%)$ & $\omega_{G E M 1}($ error $\%)$ & $\omega_{G E M 2}($ error $\%)$ & $\omega_{E B M 1}($ error $\%)$ & $\omega_{E B M 2}($ error $\%)$ \\
\hline 0.01 & 9.86969 & $9.86969(0)$ & $9.86969(0)$ & $9.86969(0)$ & $9.86969(0)$ & $9.86969(0)$ \\
0.1 & 9.87885 & $9.87885(0)$ & $9.87885(0)$ & $9.87885(0)$ & $9.87885(0)$ & $9.87885(0)$ \\
1 & 10.74956 & $10.75515(0.05200)$ & $10.75887(0.08661)$ & $10.74964(0.00074)$ & $10.75515(0.05200)$ & $10.74791(0.01535)$ \\
2 & 13.00593 & $13.05626(0.38698)$ & $13.08939(0.64171)$ & $13.00839(0.01891)$ & $13.05626(0.38698)$ & $12.99184(0.10834)$ \\
3 & 16.04479 & $16.17983(0.84164)$ & $16.26748(1.38793)$ & $16.05582(0.06875)$ & $16.17983(0.84164)$ & $16.00796(0.22954)$ \\
4 & 19.50249 & $19.73921(1.21379)$ & $19.89107(1.99246)$ & $19.52739(0.12768)$ & $19.73921(1.21379)$ & $19.43864(0.32739)$ \\
\hline
\end{tabular}

Table 2: Comparion of the approximate frequencies for C-C Microbeam

\begin{tabular}{ccccccc}
\hline$\alpha$ & $\omega_{e x}$ & $\omega_{V A}($ error $\%)$ & $\omega_{G E M 1}$ (error \%) & $\omega_{G E M 2}($ error $\%)$ & $\omega_{E B M 1}($ error $\%)$ & $\omega_{E B M 2}($ error $\%)$ \\
\hline 0.01 & 22.79293 & $22.79293(0)$ & $22.79293(0)$ & $22.79293(0)$ & $22.79293(0)$ & $22.79293(0)$ \\
0.1 & 22.79822 & $22.79822(0)$ & $22.79822(0)$ & $22.79822(0)$ & $22.79822(0)$ & $22.79822(0)$ \\
1 & 23.31999 & $23.32097(0.00420)$ & $23.32161(0.00695)$ & $23.31999(0)$ & $23.32097(0.00420)$ & $23.31969(0.00129)$ \\
2 & 24.82505 & $24.83796(0.05200)$ & $24.84655(0.08661)$ & $24.82524(0.00077)$ & $24.83796(0.05200)$ & $24.82124(0.01535)$ \\
3 & 27.128750 & $27.17877(0.18438)$ & $27.21192(0.30658)$ & $27.13030(0.00571)$ & $27.17877(0.18438)$ & $27.11442(0.05282)$ \\
4 & 30.035910 & $30.15214(0.38697)$ & $30.22867(0.64177)$ & $30.04161(0.01898)$ & $30.15214(0.38697)$ & $30.00337(0.10834)$ \\
\hline
\end{tabular}

The nonlinear frequency ratios of P-P and C-C Macrobeams are compared with those of Azraret et al. [24] for various values of the dimensionless maximum vibration amplitude $(\alpha=1,2,3,4)$, which are presented in Tables 3 and 4, respectively. Again, the accuracy of the present frequencies can be seen.
It can be seen from Tables $\mathbf{3}$ and $\mathbf{4}$ that the frequency ratio increases as the initial amplitude $\alpha$ increases, when $\alpha$ is small the linear frequency and the nonlinear frequency of the oscillatior are approximately equal.

Table 3: Comparion of the approximate frequency ratios for P-P Macrobeam

\begin{tabular}{ccccccc}
\hline$\alpha$ & Azrar et al. & VA (error \%) & GEM 1 (error \%) & GEM 2 (error \%) & EBM 1 (error \%) & EBM 2 (error \%) \\
\hline 1 & 1.0891 & $1.0897(0.0573)$ & $1.0901(0.0919)$ & $1.0891(0.0060)$ & $1.0897(0.0573)$ & $1.0889(0.0100)$ \\
2 & 1.3177 & $1.3228(0.3927)$ & $1.3262(0.6475)$ & $1.3180(0.0246)$ & $1.3228(0.3927)$ & $1.3163(0.1025)$ \\
3 & 1.6256 & $1.6393(0.8464)$ & $1.6482(1.3927)$ & $1.6267(0.0734)$ & $1.6393(0.8464)$ & $1.6219(0.2248)$ \\
4 & -------- & $2.0000(------)$ & $2.0153(-------)$ & $1.9785(-------)$ & $2.0000(------)$ & $1.9695(--------)$ \\
\hline
\end{tabular}


Time history and phase trajectory of responses for P-P and C-C microbeams, with $\alpha=1, l / h=0.25, K_{P}=10, K_{L}=40$ and $K_{N L}=50$, using various differential methods are presented in Figs. 2 and 3, respectively. High agreement between the approximate solutions can be observed.

Table 4: Comparion of the approximate frequency ratio for C-C Macrobeam

\begin{tabular}{ccccccc}
\hline$\alpha$ & Azrar et al. & VA (error \%) & GEM 1 (error \%) & GEM 2 (error \%) & EBM 1 (error \%) & EBM 2 (error \%) \\
\hline 1 & 1.0221 & $1.0231(0.1045)$ & $1.0231(0.0027)$ & $1.0231(0.1003)$ & $1.0231(0.1045)$ & $1.0231(0.0991)$ \\
2 & 1.0856 & $1.0897(0.3799)$ & $1.0901(0.0345)$ & $1.0891(0.3285)$ & $1.0897(0.3799)$ & $1.0889(0.3123)$ \\
3 & 1.1831 & $1.1924(0.7880)$ & $1.1938(0.1219)$ & $1.1902(0.6083)$ & $1.1924(0.7880)$ & $1.1896(0.5494)$ \\
4 & 1.3064 & $1.3228(1.2611)$ & $1.3262(0.2538)$ & $1.3180(0.8899)$ & $1.3228(1.2611)$ & $1.3163(0.7615)$ \\
\hline
\end{tabular}
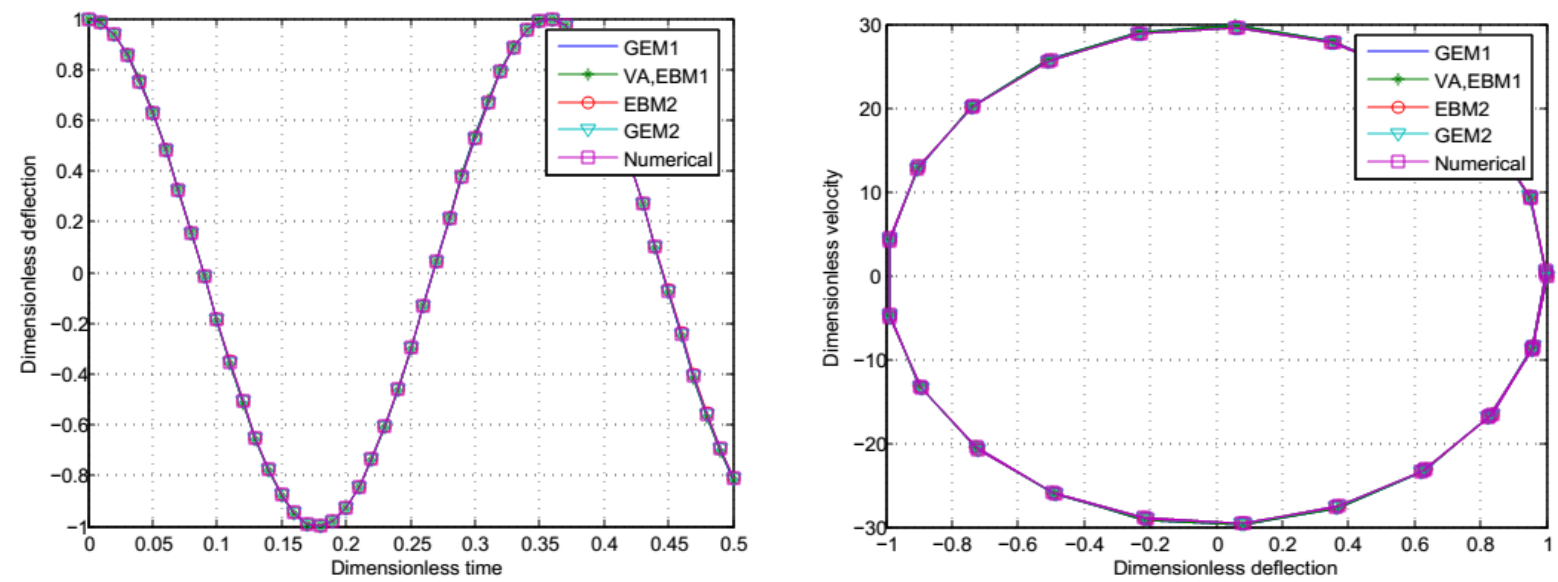

Fig. 2: Time history and phase trajectory of response for P-P microbeam
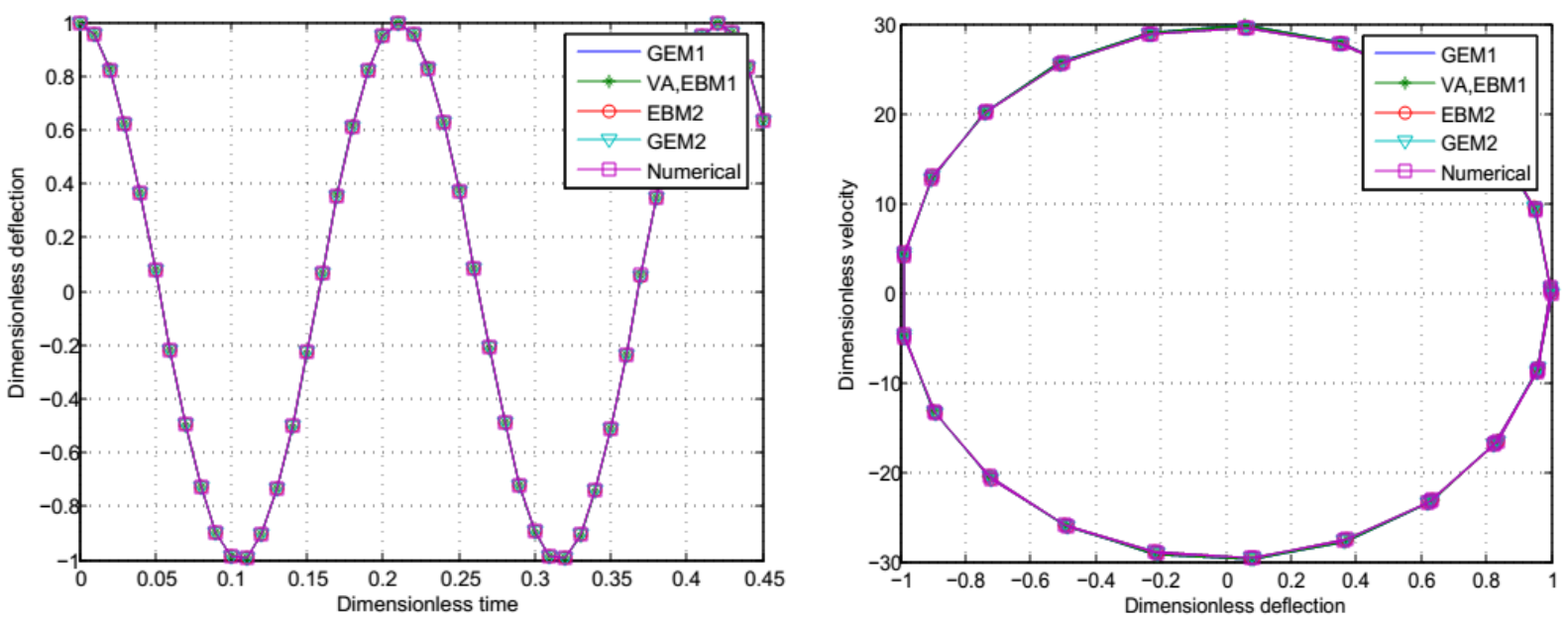

Fig. 3: Time history and phase trajectory of response for C-C microbeam

The effects of the initial amplitude $\alpha$ on the nonlinear frequency ratio $\left(\omega_{N L} / \omega_{L}\right)$ and the nonlinear frequency $\omega_{N L}$ of microbeams based on the dimensionless material length scale parameter $(l / h)$ are plotted in Figs. 4 and 5. When the dimensionless material length scale parameter increases, then the frequency ratio decreases but the nonlinear frequency increases. 


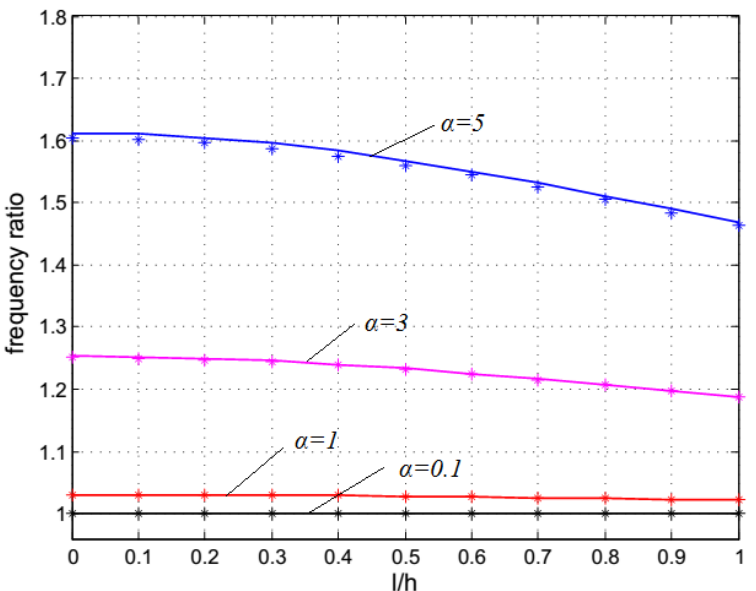

(a)

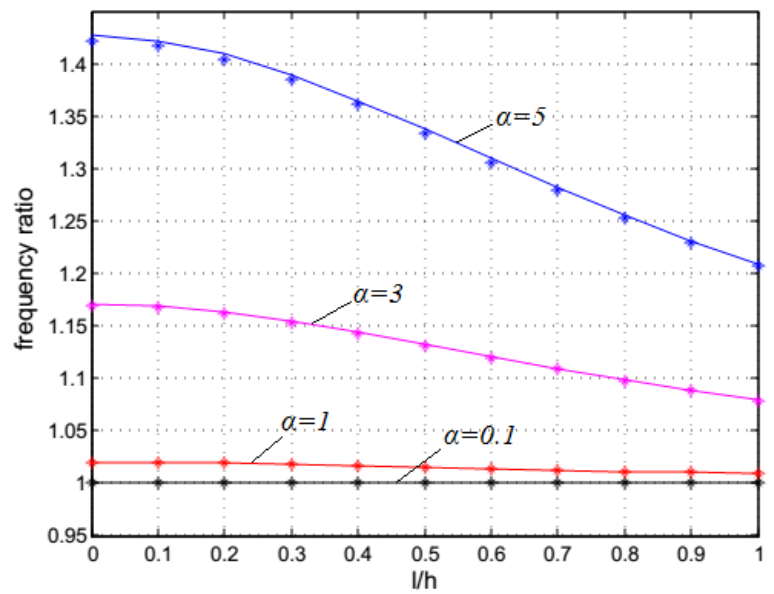

(b)

Fig. 4: The effect of the initial amplitude on the frequency ratio of microbeams based on the dimensionless material length scale parameter with $K_{P}=100, K_{L}=100$ and $K_{N L}=100$; (a) P-P microbeam, (b) C-C microbeam; ( * : VA and EBM1, - : GEM1)

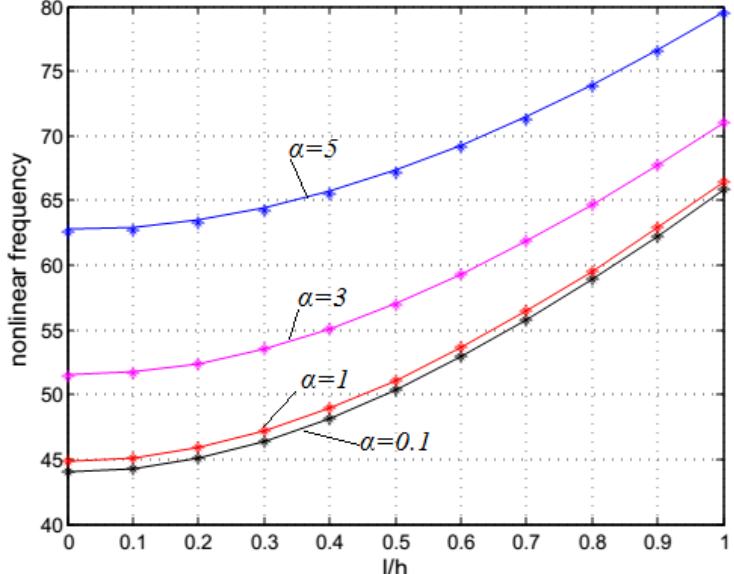

(a)

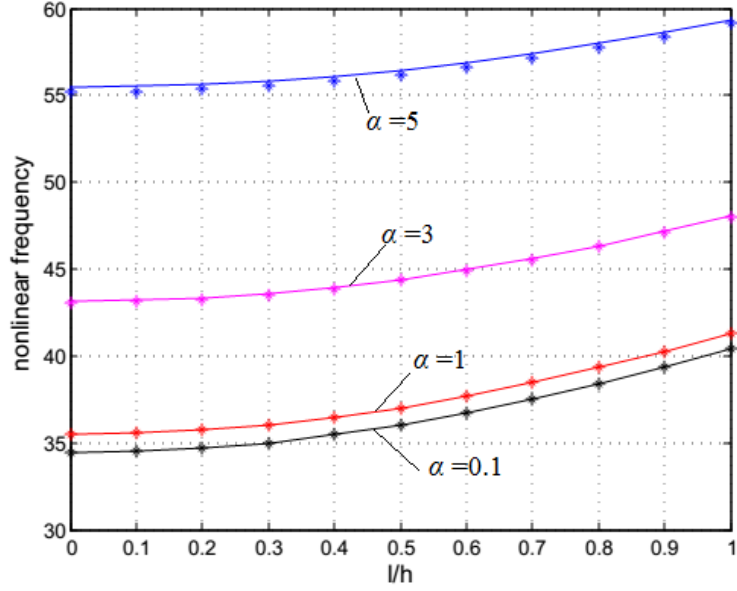

(b)

Fig. 5: The effect of the initial amplitude on the nonlinear frequency of microbeams based on the dimensionless material length scale parameter with $K_{P}=100, K_{L}=100$ and $K_{N L}=100$; (a) P-P microbeam, (b) C-C microbeam; ( * : VA and EBM1, - : GEM1)

The variations of the nonlinear frequency ratio and the nonlinear frequency with the initial amplitude for various values of the dimensionless length scale parameter are investigated and showed

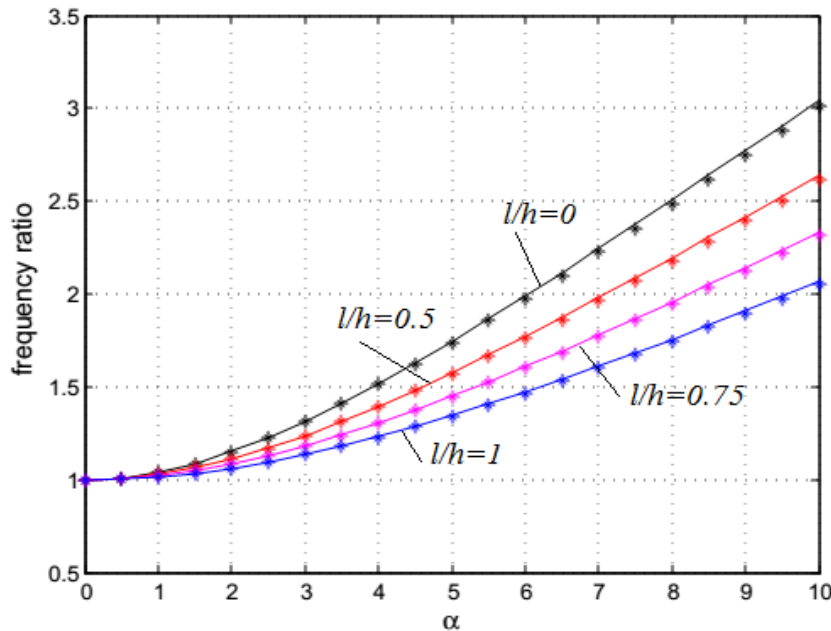

(a) in Figs. 6 and 7. We see that the frequency ratio and the nonlinear frequency of microbeams increase when the dimensionless amplitude increses.

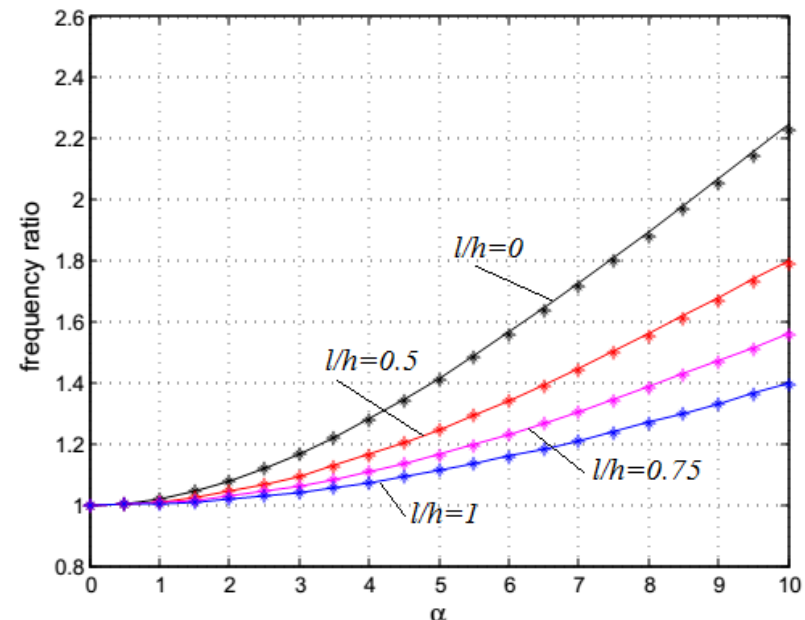

(b)

Fig. 6: The variation of the frequency ratio of microbeams with the dimensionless amplitude for various values of the dimensionless material length scale parameter with $K_{P}=10, K_{L}=100$ and $K_{N L}=10$; (a) P-P microbeam, (b) C-C microbeam; ( * : VA and EBM1, - : GEM1) 


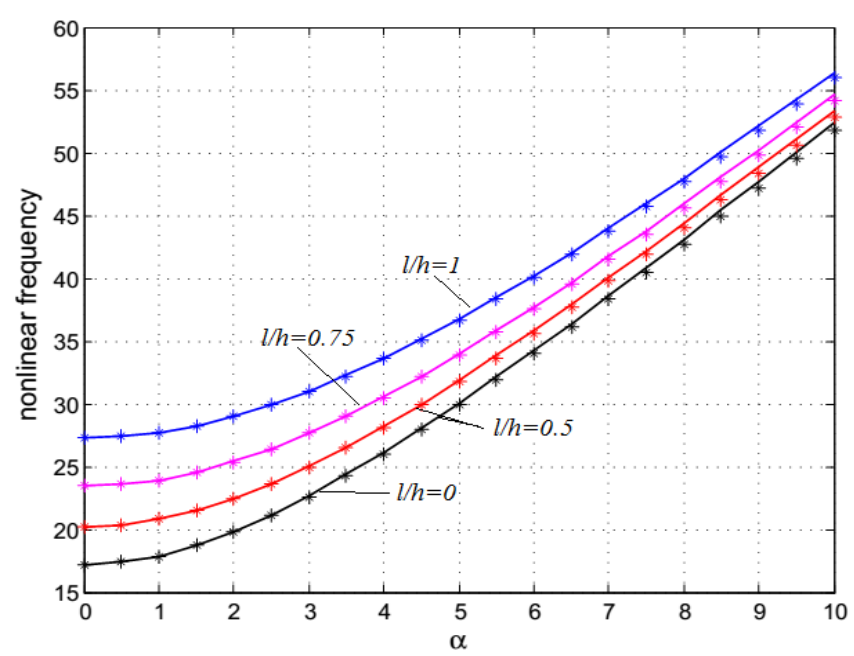

(a)

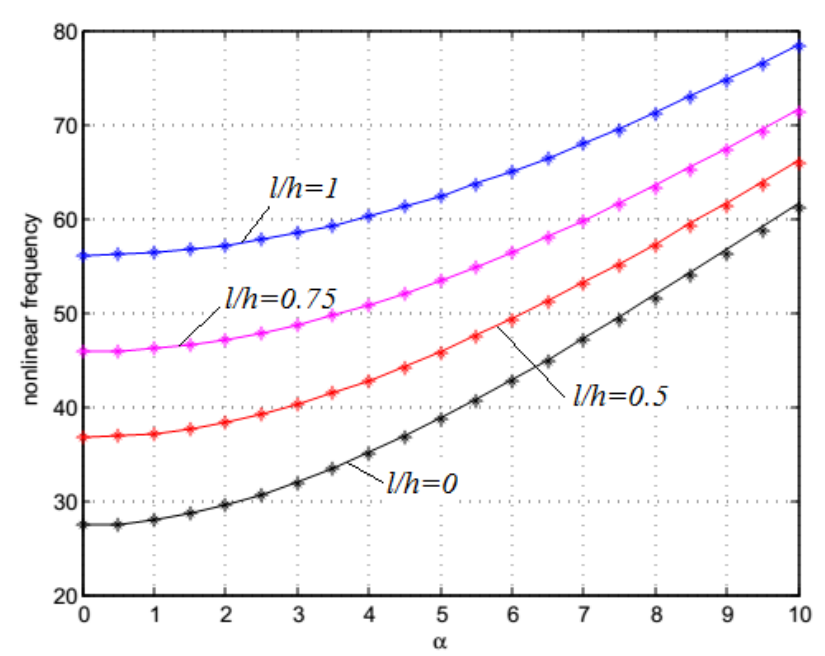

(b)

Fig. 7: The variation of the nonlinear frequency of microbeams with the dimensionless amplitude for various values of the dimensionless material length scale parameter with $K_{P}=10, K_{L}=100$ and $K_{N L}=10$; (a) P-P microbeam, (b) C-C microbeam; ( * VA and EBM1, - : GEM1)

Comparisons of the effect of the coefficients of the nonlinear foundation on the vibration behavior of the microbeams using various methods are also investigated. First, the variations of the nonlinear frequency ratio and the nonlinear frequency with the Winkler parameter for various values of the dimensionless length

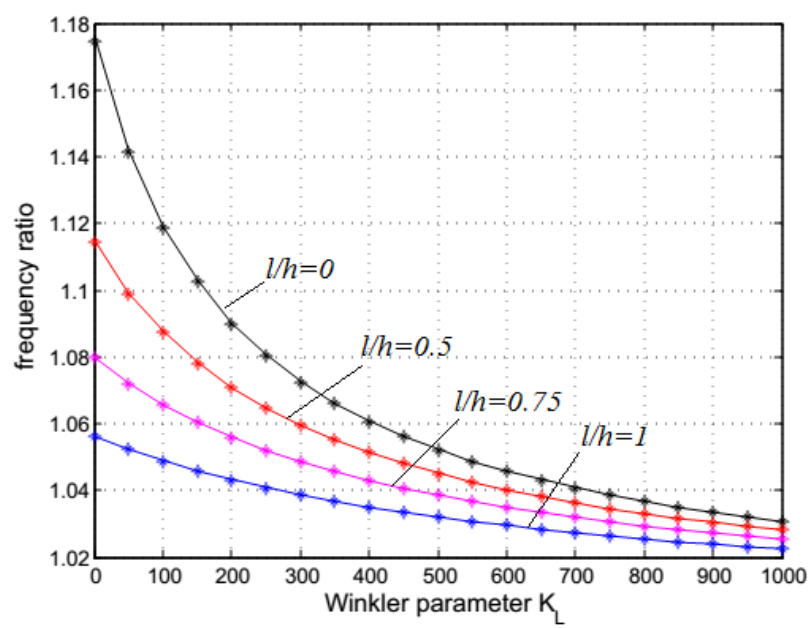

(a) scale parameter are showed in Figs. 8 and $\mathbf{9}$. We see that when the dimensionless amplitude increases, then the frequency ratio of microbeams decreases but the nonlinear frequency of microbeams increases.

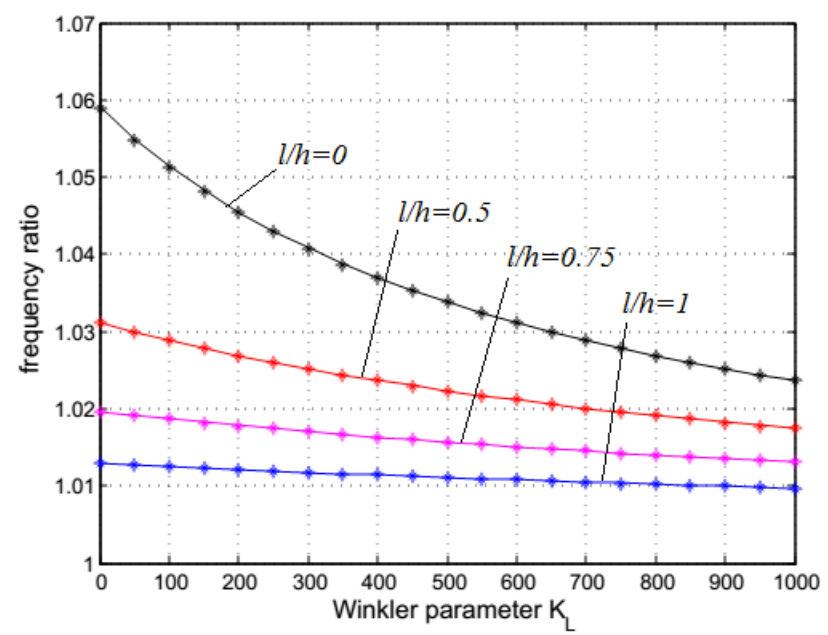

(b)

Fig. 8: The variation of the frequency ratio of microbeams with the Winkler parameter for various values of the dimensionless material length scale parameter with $K_{P}=10, K_{N L}=100$ and $\alpha=1$; (a) P-P microbeam, (b) C-C microbeam; ( * : VA and EBM1, - : GEM1)

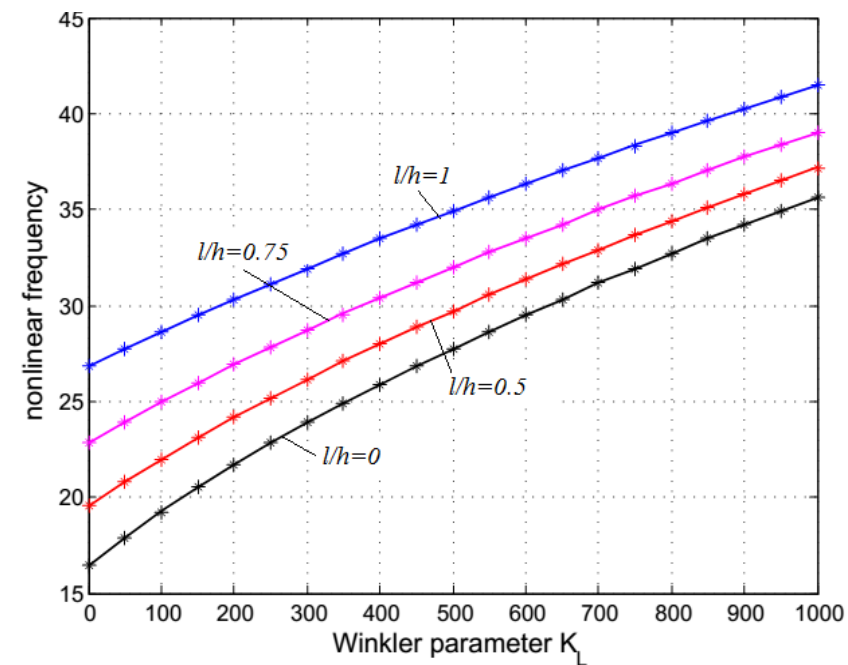

(a)

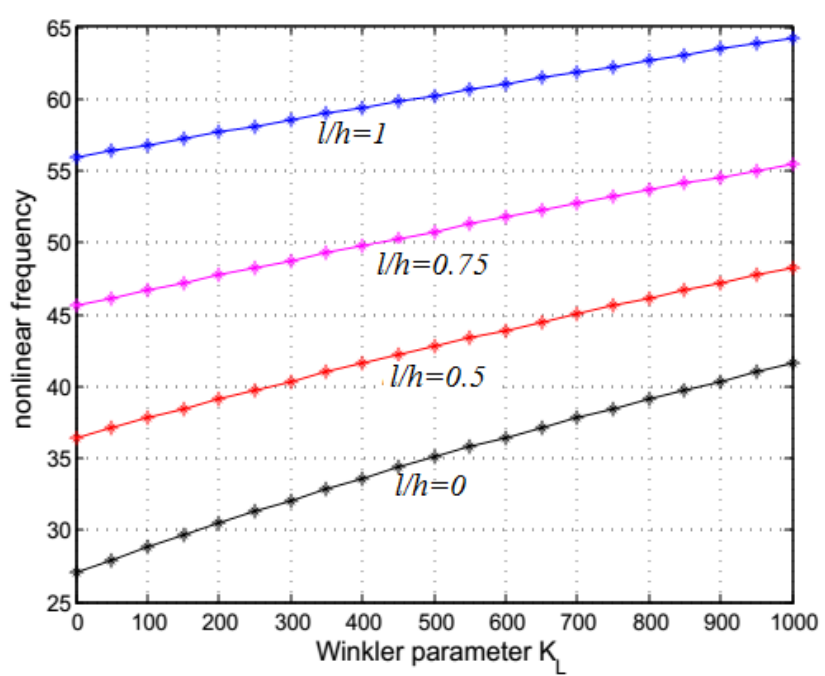

(b)

Fig. 9: The variation of the nonlinear frequency of microbeams with the Winkler parameter for various values of the dimensionless material length scale parameter with $K_{P}=10, K_{N L}=100$ and $\alpha=1$; (a) P-P microbeam, (b) C-C microbeam; (* : VA and EBM1, - : GEM1) 
Second, the variations of the nonlinear frequency ratio and the nonlinear frequency with the Pasternak parameter for various values of the dimensionless length scale parameter are presented in Figs. 10 and 11. As the same as the effect of the Winkler parame-

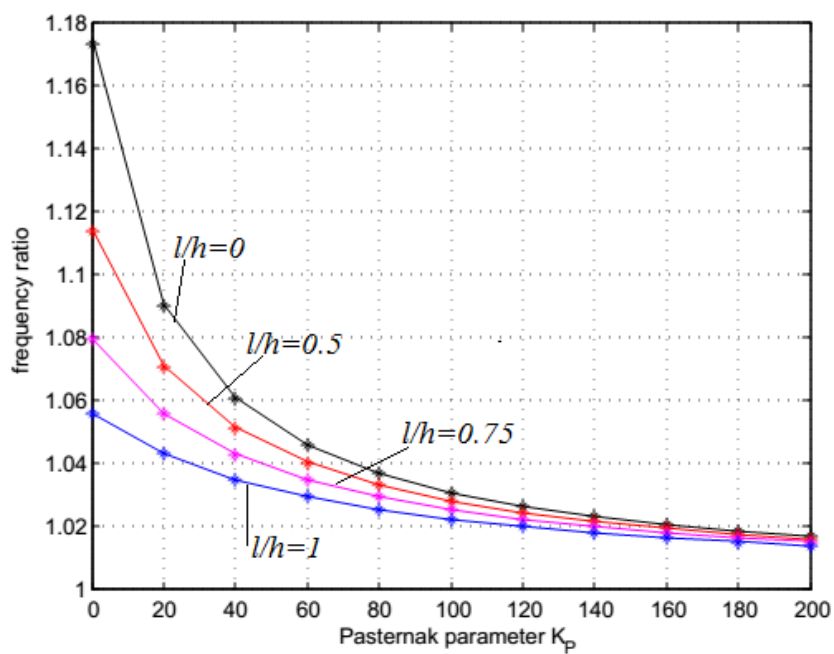

(a)

Fig. 10: The variation of the frequency ratio of microbeams with the Paternak parameter for various values of the dimensionless material length scale parameter with $K_{L}=100, K_{N L}=100$ and $\alpha=1$; (a) P-P microbeam, (b) C-C microbeam; ( * : VA and EBM1, - : GEM1)

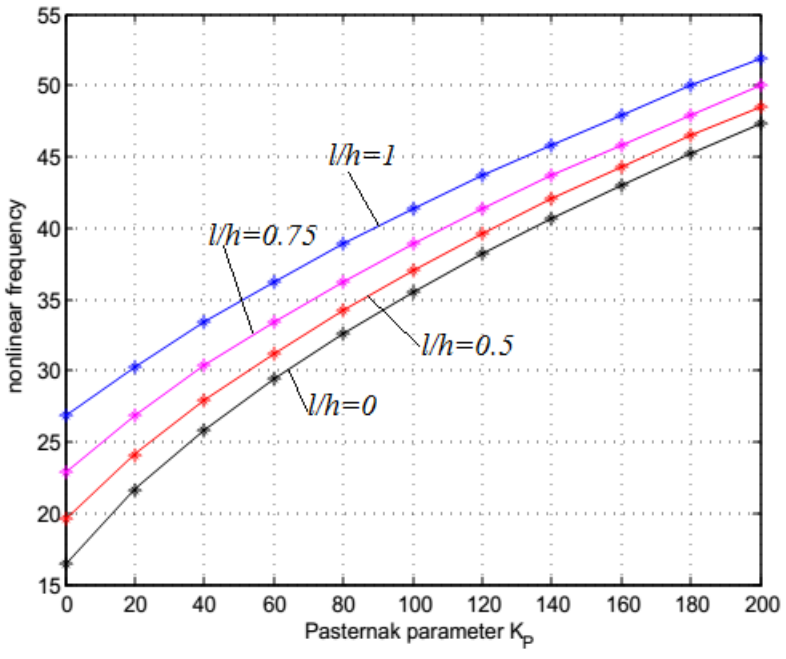

(a) ter on the vibration behavior of the microbeams, when the dimensionless amplitude increases, then the frequency ratio of microbeams decreases but the nonlinear frequency of microbeams increases.

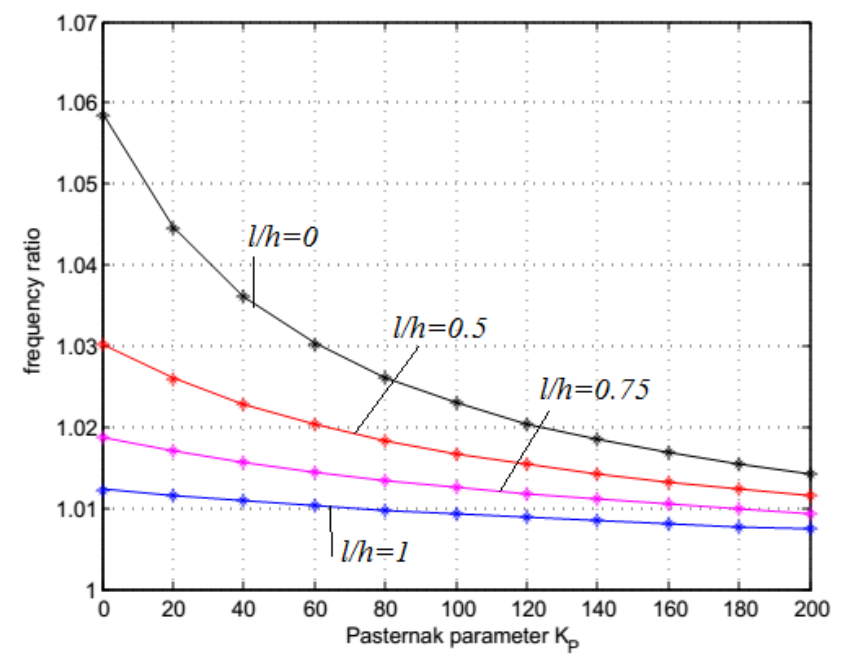

(b)

Fig. 11: The variation of the nonlinear frequency of microbeams with the Paternak parameter for various values of the dimensionless material length scale parameter with $K_{L}=100, K_{N L}=100$ and $\alpha=1$; (a) P-P microbeam, (b) C-C microbeam; ( * : VA and EBM1, - : GEM1)

And last, the variations of the nonlinear frequency ratio and the nonlinear frequency with the nonlinear foundation parameter for various values of the dimensionless length scale parameter are investigated and presented in Figs. 12 and 13. It is not the same as the effect of the Winkler parameter and the Pasternak parameter on the vibration behavior of the microbeams, when the dimensionless amplitude increases, then all of the frequency ratio of microbeams and the nonlinear frequency of microbeams increase. 


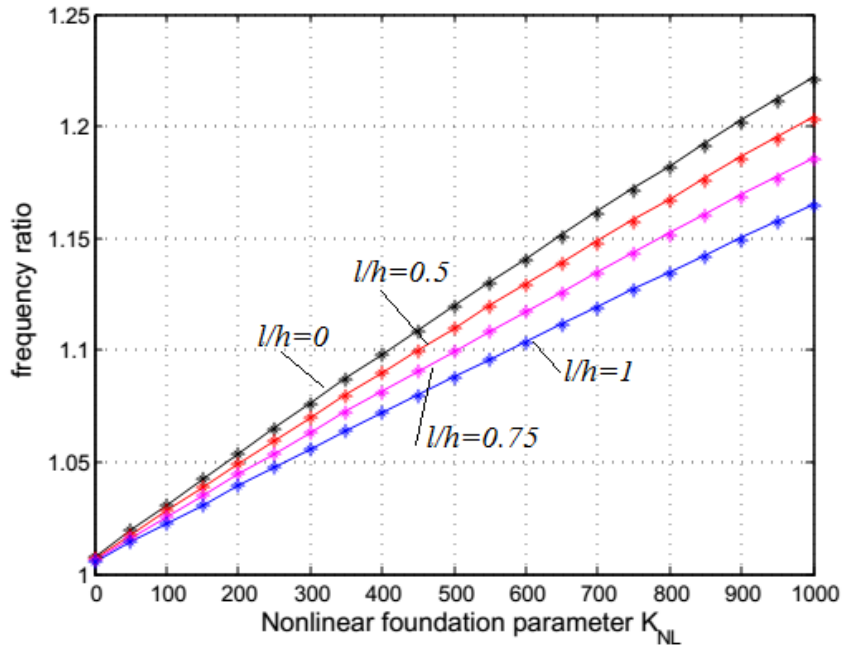

(a)

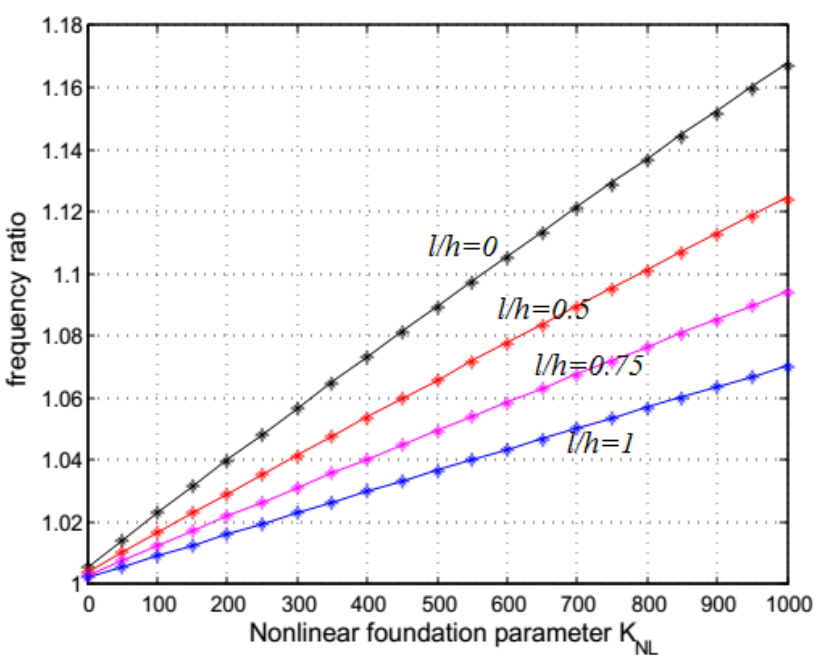

(b)

Fig. 12: The variation of the frequency ratio of microbeams with nonlinear foundation parameter for various values of the dimensionless material length scale parameter with $K_{P}=100, K_{L}=100$ and $\alpha=1$; (a) P-P microbeam, (b) C-C microbeam; ( * : VA and EBM1, - : GEM1)

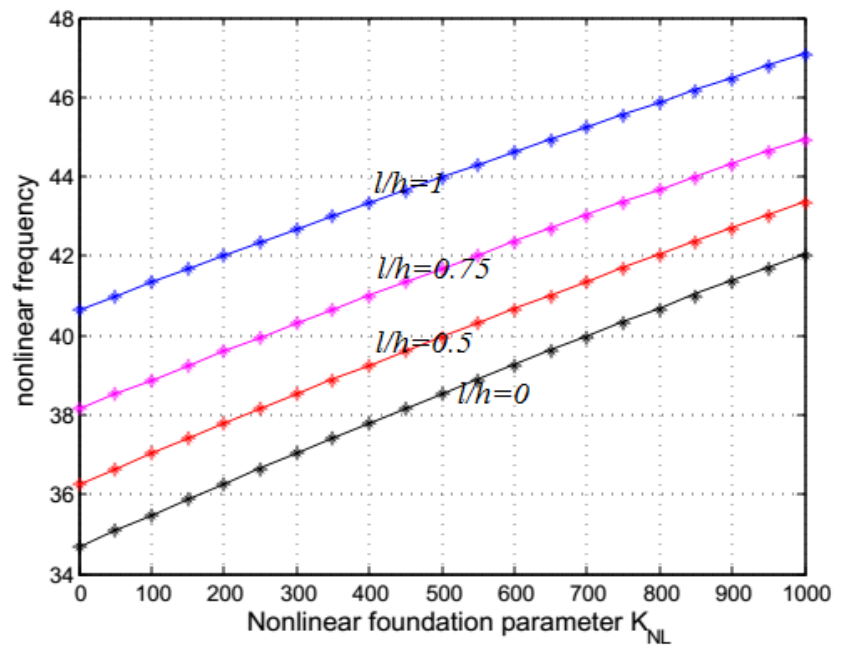

(a)

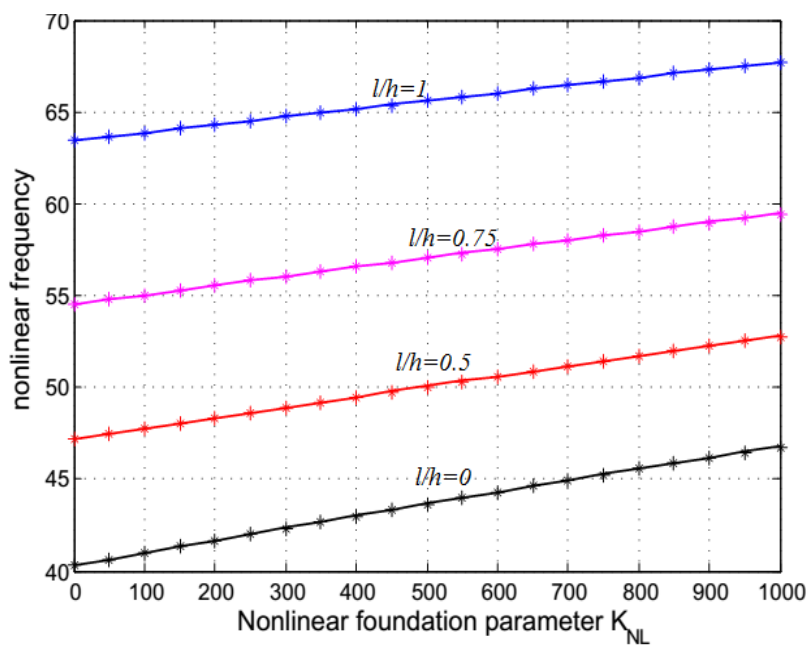

(b)

Fig. 13: The variation of the nonlinear frequency of microbeams with nonlinear foundation parameter for various values of the dimensionless material length scale parameter with $K_{P}=100, K_{L}=100$ and $\alpha=1$; (a) P-P microbeam, (b) C-C microbeam; ( * VA and EBM1, - : GEM1)

\section{Conclusion}

In this work, nonlinear free vibration of microbeams based on nonliear elastic foundation with three layers is intestigated using the high-order Energy Balance method and the high-order Global Error Minimization method. The accuracy of approximate solutions has been improved by using the second-order approximation. The frequency-amplitude relationships of microbeams with P-P and $\mathrm{C}-\mathrm{C}$ end conditions are obtained in closed-forms. The present solutions are compared with the previous published ones. Comparisons show the accuracy of the obtained solutions. The effects of the initial amplitude, the dimensionless material length scale parameter and the coefficients of the nonlinear foundation on the vibration behavior of the microbeams are investigated.

\section{Acknowledgement}

This work is supported by Thai Nguyen University of Technology for a scientific project.

\section{Appendix}

For the osccillator given in the form:

$$
\ddot{X}+a_{1} X+a_{2} X^{3}=0, X(0)=A, \dot{X}(0)=0
$$

The exact frequency is:

$$
\omega_{e x}=\frac{2 \pi}{4 \sqrt{2} \int_{0}^{\pi / 2} \frac{d t}{\sqrt{a_{2} A^{2} \cos ^{2}(t)+a_{2} A^{2}+2 a_{1}}}}
$$

\section{References}

[1] Senturia, S. D., Microsystem design. Boston: Kluwer Academic Publishers, (2001).

[2] McFarland, A. W., and Colton, J. S., "Role of material microstructure in plate stiffness with relevance to microcantilever sensors". Journal of Micromechanical and Microengineering, Vol.15 (2005), pp. 1060-1067.

[3] Toupin, R. A., "Elastic materials with couple stresses. Archives of Rational of Mechanical and Analysis", Vol. 11(1962), pp. 385-414.

[4] Mindlin R. D., Tiersten H. F., "Effects of couple-stresses in linear elasticity". Archive for Rational Mechanics and Analysis; Vol.11(1962), pp.415-48.

[5] Mindlin R. D., "Influence of couple-stresses on stress concentrations". Experimental Mechanics; Vol. 3(1963), pp.1-7.

[6] Aifantis E. C., "Strain gradient interpretation of size effects". International Journal of Fracture; Vol. 95(1999), pp.1-4.

[7] Eringen A. C., "Theory of micropolar plates". Zeitschrift für angewandte Mathematik und Physik;Vol.18(1967), pp.12-30. 
[8] Eringen A. C., "Nonlocal polar elastic continua”. International Journal of Engineering Science; Vol.10(1972), pp. 1-16.

[9] Gurtin M. E., Weissmuller J., Larche F., "The general theory of curved deformable interfaces in solids at equilibrium". Philosophical Magazine A (1998); pp. 1093-109.

[10] Yang, F., Chong, A. C. M., Lam, D. C. C., \& Tong, P., "Couple stress based strain gradient theory for elasticity". International Journal of Solids and Structures, Vol.39(2002),pp. 2731-2743.

[11] Ma H. M., Gao X. L., Reddy J. N., "A microstructure-dependent Timoshenko beam model based on a modified couple stress theory". Journal of the Mechanics and Physics of Solids; Vol.56(2008), pp.3379-91.

[12] Kong S., Zhou S., Nie Z., Wang K., "The size-dependent natural frequency of Bernoulli-Euler micro-beams". International Journal of Engineering Science; Vol. 46(2008), pp.427-37.

[13] Ma H. M., Gao X. L., Reddy J. N., "A nonclassical ReddyLevinson beam model based on a modified couple stress theory". International Journal for Multiscale Computational Engineering; Vol.8(2010), pp. 167-80.

[14] Wang B., Zhao J., Zhou S., “A micro scale Timoshenko beam model based on strain gradient elasticity theory". European Journal of Mechanics - A/Solids; Vol.29(2010), pp.591-9.

[15] Wang L., "Size-dependent vibration characteristics of fluidconveying microtubes". Journal of Fluids and Structures; Vol.26(2010), pp.675-84.

[16] Simsek M., "Dynamic analysis of an embedded microbeam carrying a moving microparticle based on the modified couple stress theory". International Journal of Engineering Science; Vol.48(2010), pp.1721-32.

[17] Simsek M., Kocatürk T., Akbas S. D., "Static bending of a functionally graded microscale Timoshenko beam based on the modified couple stress theory". Composite Structures; Vol. 95(2013) pp.740-7.

[18] Simsek M., "Nonlinear static and free vibration analysis of microbeams based on the nonlinear elastic foundation using modified couple stress theory and He's variational method". Composite Structures; Vol.112(2014), pp. 264-272.

[19] J. H. He, "Preliminary report on the energy balance for nonlinear oscillations". Mechanics Research Communications, 29(23):107-111

[20] J. H. He, "Variational approach for nonlinear oscillators. Chaos", Solitons Fractals; Vol.34(2007), pp. 1430-9.

[21] J. H. He., "Hamiltonian approach to nonlinear oscillators". Physics Letters A.Vol.374(2010), pp. 2312-2314.

[22] Yadollah Farzaneh, Ali Akbarzadeh Tootoonchi. "Global Error Minimization method for solving strongly nonlinear oscillator differential equations". Computers and Mathematics with Applications, Vol.59 (2010), pp. 2887-2895.

[23] Seher Durmaz and Metin Orhan Kaya. "High-Order Energy Balance Method to Nonlinear Oscillators". Journal of Applied Mathematics, Vol. 2012(2012).

[24] Azrar L., Benamar R., White R. G., "A semi-analytical approach to the nonlinear dynamic response problem of $\mathrm{S}-\mathrm{S}$ and $\mathrm{C}-\mathrm{C}$ beams at large vibration amplitudes. Part I: General theory and application to the single mode approach to free and forced vibration analysis". Journal of Sound and Vibration; Vol.224(1999), pp.183-207. 